\title{
Erratum: Microscopic formulation of dynamical spin injection in ferromagnetic-nonmagnetic heterostructures [Phys. Rev. B 96, 035420 (2017)]
}

Amin Ahmadi and Eduardo R. Mucciolo

(Q) (Received 29 June 2018; published 13 July 2018)

DOI: 10.1103/PhysRevB.98.039902

In the description of Fig. 5 , in the first paragraph on the right column on p. 10, the correct decay lengths are $4.5 \times 10^{3}, 2.7 \times 10^{3}$, and $2.4 \times 10^{3}$ lattice units. The factor of $10^{3}$ is missing in the original text. However the units that are shown in the labels of the plot in Fig. 5 are correct. 